\title{
On the Leading Error Term of Exponentially Fitted Numerov Methods
}

\author{
D. Hollevoet, M. Van Daele and G. Vanden Berghe \\ Vakgroep Toegepaste Wiskunde en Informatica, Ghent University, \\ Krijgslaan 281-S9, B-9000 Gent, Belgium
}

\begin{abstract}
Second-order boundary value problems are solved with exponentially-fitted Numerov methods. In order to attribute a value to the free parameter in such a method, we look at the leading term of the local truncation error. By solving the problem in two phases, a value for this parameter can be found such that the tuned method behaves like a sixth order method. Furthermore, guidelines to choose between multiple possible values for this parameter are given.
\end{abstract}

Keywords: Numerov methods, Boundary value problems, Exponential fitting, Error term, Parameter selection PACS: $02.60 . \mathrm{Lj}$

\section{INTRODUCTION}

The Numerov method, also known as Cowell's method, is a well-known method for solving second order differential equations. We will apply this method to boundary value problems of the form

$$
y^{\prime \prime}=f(t, y), \quad f(a)=\alpha, \quad f(b)=\beta .
$$

If the solution of such a problem has an exponential or trigonometric character, Numerov's method, whose construction relies on polynomials, may not be very well-suited. Exponentially fitted [1] variants of this method may be more fit for the task.

For $N \geq 3$, we define $t_{j}:=a+j h$ for $j=0,1, \ldots, N+1$ and $h:=(b-a) /(N+1)$. The approximate value of the solution $y\left(t_{j}\right)$ will be denoted as $y_{j}$.

\section{CONSTRUCTION OF EXPONENTIALLY FITTED METHODS}

The main idea of exponential fitting is to extend the fitting space of a method beyond purely polynomial to a combination of polynomials and exponentials, a so-called hybrid set. Given the value of $M$, the maximal number of independent functions in the fitting space of the method, one can construct methods with fitting spaces of the form

$$
\left\{1, t, t^{2}, \ldots, t^{K}\right\} \cup\left\{e^{ \pm \mu t}, t e^{ \pm \mu t}, t^{2} e^{ \pm \mu t}, \ldots, t^{P} e^{ \pm \mu t}\right\}
$$

with $P$ and $K$ satisfying $M-3=2 P+K$.

The construction itself is done by means of the six-step procedure by Ixaru and Vanden Berge [1]. For every possible combination of $K$ en $P$ adhering the constraint ut supra, this procedure gives a set of weight functions depending on $\mu$ and $h$, to use in the finite difference scheme.

In particular, we will consider methods of the form

$$
y_{j-1}+a_{0}(\mu, h) y_{j}+y_{j+1}=h^{2}\left(b_{1}(\mu, h) f_{j-1}+b_{0}(\mu, h) f_{j}+b_{1}(\mu, h) f_{j+1}\right) .
$$

In this case, $M=6$, which allows four possible combinations of $K$ and $P$ :

- $P=-1, \quad K=5, \quad F S=\left\{1, t, t^{2}, t^{3}, t^{4}, t^{5}\right\}$ (Numerov's method)

- $P=0, \quad K=3, \quad F S=\left\{1, t, t^{2}, t^{3}, e^{ \pm \mu t}\right\}$

- $P=1, \quad K=1, \quad F S=\left\{1, t, e^{ \pm \mu t}, t e^{ \pm \mu t}\right\}$

$\cdot P=2, \quad K=-1, \quad F S=\left\{e^{ \pm \mu t}, t e^{ \pm \mu t}, t^{2} e^{ \pm \mu t}\right\}$

CP1048, Numerical Analysis and Applied Mathematics, International Conference 2008

edited by T. E. Simos, G. Psihoyios, and Ch. Tsitouras

(C) 2008 American Institute of Physics 978-0-7354-0576-9/08/ $\$ 23.00$ 
The coefficient functions for the exponentially fitted methods have already been established in [1, 3]. For the remainder of the discussion, we are especially interested in the form of the local truncation error of these modified Numerov methods. Instead of following the approach in [2] to construct this 1te in a closed form, we will focus on the leading error term.

As already stated in [4], the expressions for the lte of the exponentially fitted Numerov methods are given by

$$
\begin{aligned}
& \cdot P=0 \\
& \text { lt } e_{0}=h^{6} \phi_{0}(Z) \mathbf{D}^{4}\left(\mathbf{D}^{2}-\mu^{2}\right)[\mathbf{y}]+\mathscr{O}\left(h^{8}\right) \\
& \cdot P=1 \\
& \text { lte }_{1}=h^{6} \phi_{1}(Z) \mathbf{D}^{2}\left(\mathbf{D}^{2}-\mu^{2}\right)^{2}[\mathbf{y}]+\mathscr{O}\left(h^{8}\right) \\
& \cdot P=2 \\
& \\
& \text { lte } e_{2}=h^{6} \phi_{2}(Z)\left(\mathbf{D}^{2}-\mu^{2}\right)^{3}[\mathbf{y}]+\mathscr{O}\left(h^{8}\right) .
\end{aligned}
$$

\section{FREQUENCY ESTIMATION AND SELECTION}

\section{Annihilating the plte by local tuning}

When solving a boundary value problem with an exponentially fitted method, one encounters a system of equations of the form (1) with $j=1,2 \ldots N$. As shown above, the difference between the exact solution $y\left(t_{j}\right)$ and $y_{j}(\mu)$ begins with $h^{6} \phi_{P} D^{K+1}\left(D^{2}-\mu^{2}\right)^{P+1}[y]\left(t_{j}\right)$. If $\mu$ is set to a value $\mu_{j}$ such that $D^{K+1}\left(D^{2}-\mu_{j}^{2}\right)^{P+1}[y]\left(t_{j}\right)=0$, then the plte is annihilated and the obtained locally tuned method will behave like a 6 th order method.

In order to determine these plte annihilating $\mu_{j}$, one has to solve

$$
D^{K+1}\left(D^{2}-\mu_{j}^{2}\right)^{P+1}[y]\left(t_{j}\right)=0
$$

for $j=1,2 \ldots N$.

In particular, for the exponentially fitted Numerov methods we are considering, these expressions are

$$
\begin{array}{ll}
P=0, & D^{4}\left(D^{2}-\mu^{2}\right)[y]\left(t_{j}\right)=y^{(6)}\left(t_{j}\right)-\mu^{2} y^{(4)}\left(t_{j}\right)=0 \\
P=1, & D^{2}\left(D^{2}-\mu^{2}\right)^{2}[y]\left(t_{j}\right)=y^{(6)}\left(t_{j}\right)-2 \mu^{2} y^{(4)}\left(t_{j}\right)+\mu^{4} y^{(2)}\left(t_{j}\right)=0 \\
P=2, & \left(D^{2}-\mu^{2}\right)^{3}[y]\left(t_{j}\right)=y^{(6)}\left(t_{j}\right)-3 \mu^{2} y^{(4)}\left(t_{j}\right)+3 \mu^{4} y^{(2)}\left(t_{j}\right)-\mu^{6} y\left(t_{j}\right)=0
\end{array}
$$

The dependency of each of these expressions on higher derivatives of $y$ can be mitigated by rewriting those derivatives in terms of $f(t, y(t))$ and its derivatives. As a result, only $y$ and $y^{\prime}$ are needed to solve equation (3), (4) and (5).

To cope with this circular dependency, one can solve the problem in two phases:

- The solution of the problem is approximated with the classical method. This results in a series of points $y_{j}$, which is then used to approximate $y^{\prime}$ in $t_{j}$. This can be done with standard finite difference formulae. Now (2) can be solved for $\mu_{j}$ using these approximations for $y$ and $y^{\prime}$.

- The problem is solved again, using an exponentially fitted method obtained by tuning equation (1) to the local frequency $\mu_{j}$ for $j=1,2 \ldots N$.

\section{Frequency selection}

For $P=0$, the equation that should be solved to find a plte annihilating frequency $\mu_{j}$, is quadratic in $\mu^{2}$. There is however only one meaningfull solution, since $\mu$ and $-\mu$ are mapped upon the same frequency by the antisymmetry in $t^{P} e^{ \pm \mu t}$. Starting from $P>0$, there can be multiple plte annihilating frequencies. Which frequency should be chosen?

If an exponentially fitted method can be tuned such that the solution of the problem at hand falls within the fitting space of that method, then (2) will exhibit a constant (i.e. time-independent) solution. Tuning this method will not only annihilate the leading error term, but the entire truncation error. It can be proven that when a method with $P=P_{0}$ 

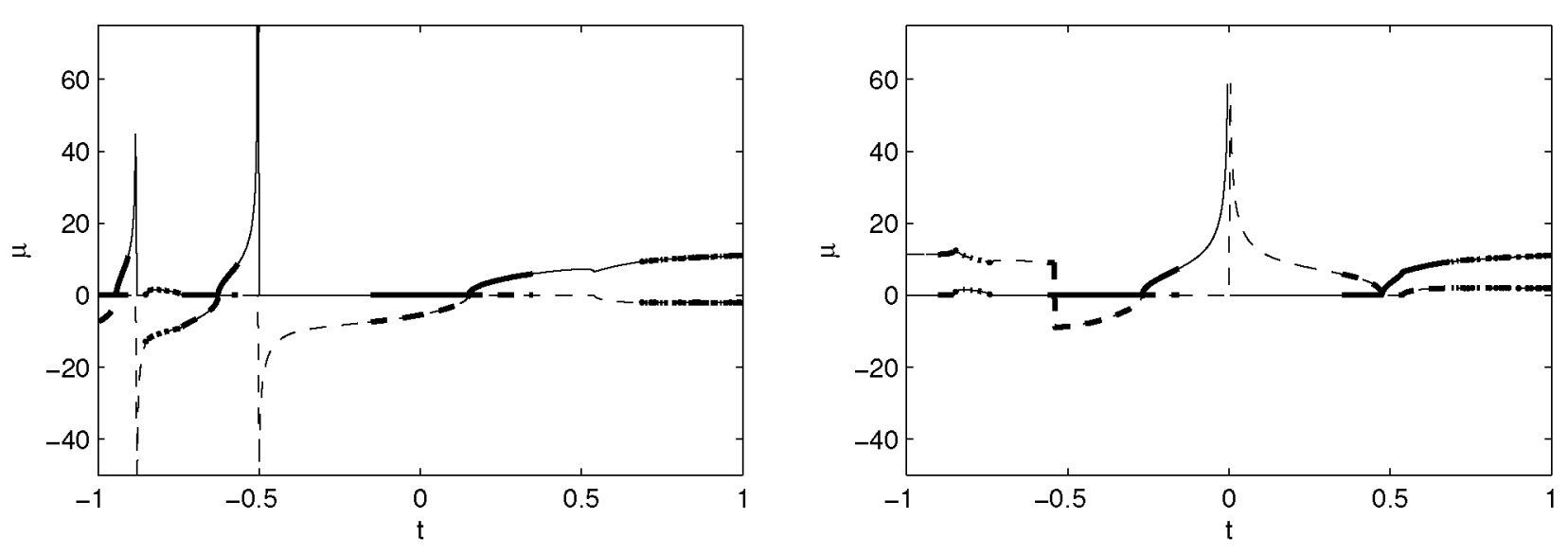

FIGURE 1. The two solutions $\mu_{j 1}$ (left) and $\mu_{j 2}$ (right) of (4), applied to the approximated solution of Example 1 for $h=1 / 256$. Solid lines represent the real part of the solution, dashed lines show the imaginary component. Both $\mu_{j 1}$ and $\mu_{j 2}$ tend to infinity at some point. In order to avoid these peaks, one could take $\min \left(\left|\mu_{j 1}\right|,\left|\mu_{j 2}\right|\right)$ at every point, as indicated by a thicker line.

can solve such a problem up to machine accuracy by tuning it to $\mu_{c}$, equation (2) associated with a method with $P=P_{n}>P_{0}$ will feature the same solution $\mu_{c}$, with multiplicity at least $P_{n}-P_{0}+1$.

In general however, equation (2) gives rise to $P+1$ time-dependent solutions. It is quite common that when solving (2), rather large frequencies are found, due to a denominator in the expression for the solution tending towards zero. These frequencies should be avoided when possible.

One simple strategy is to select $\min \left\{\left|\mu_{j i}\right|, i=1 \ldots P+1\right\}$ for $j=1,2 \ldots N$. This avoids large solutions in favour of smaller and likely more well-behaved frequencies.

\section{NUMERICAL RESULTS}

We will look at the results obtained when the ideas above are applied to the following problem, taken from [5] by Cash:

$$
\varepsilon y^{\prime \prime}-t y=0, \quad y(-1)=1, \quad y(1)=1
$$

The analytical solution is given by $y(t)=A \mathrm{~A}_{\mathrm{i}}\left(t \varepsilon^{-1 / 3}\right)+B \mathrm{~B}_{\mathrm{i}}\left(t \varepsilon^{-1 / 3}\right)$, a combination of Airy functions, with $A$ and $B$ constants depending on $\varepsilon$. We will solve this problem for $\varepsilon=1 / 100$.

We immediately start with the method $P=1$, because (4) can have two solutions at every point. This allows us to avoid the large peaks in both solutions $\mu_{j 1}$ and $\mu_{j 2}$ shown in Fig. 1 by taking the minimum (in norm) of both in every point (indicated by a thicker line).

This was done for several stepsizes and the result is shown in Fig. 2 . As can be seen, the strategy to take $\min \left(\mu_{j 1}, \mu_{j 2}\right)$ as frequency to tune our method to, pays off, as it leads to a method that behaves like 6 th order method. If there were no possibility to choose between $\mu_{j 1}$ and $\mu_{j 2}$, as would be with a method for which $P=0$, the result would be suboptimal and even worse than classical for $\mu_{j 1}$ and larger stepsizes.

\section{CONCLUSION}

We have looked into the problem of finding a frequency to tune an exponentially fitted method. The objective was to gain accuracy when solving a boundary value problem. In order to achieve this, we looked at the form of the leading term of the local truncation error. By solving the problem in two phases, these expressions can be solved for the frequency that annihilates them. A method that is tuned to these frequencies behaves like a 6th order method.

Furthermore, guidelines were given to select a good frequency when several possibilities are available. If a timeindependent plte annihilating frequency is available, this one should be preferred. If not, larger frequencies should be avoided, for example by taking the smallest in norm in every knot point. 


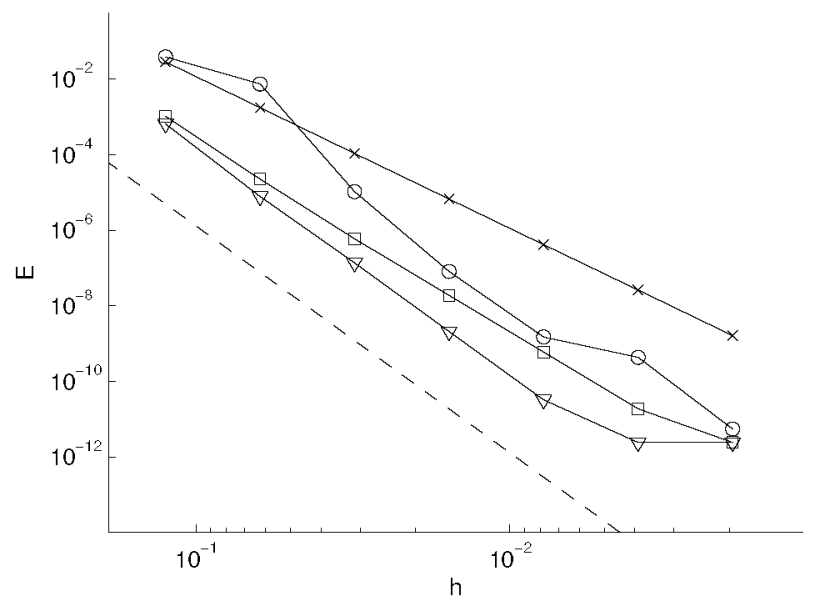

FIGURE 2. The maximum error for: classical Numerov $[\times], P=1$ tuned to $\mu_{j 1}[\circ], P=1$ tuned to $\mu_{j 2}[\square]$ and $P=1$ tuned to $\min \left(\mu_{j 1}, \mu_{j 2}\right)[\nabla]$. The ability to choose between $\mu_{j 1}$ and $\mu_{j 2}$ is clearly an advantage when solving this problem, since it leads to better results: the last line mentioned lies parallel to the dashed line, indicating a 6 th order behaviour.

It should be noted that these remarks can also be applied to other methods for solving differential equations, as well as to other types of problems.

\section{REFERENCES}

1. L. Gr. Ixaru and G. Vanden Berghe, Exponential fitting, (Kluwer Academic Publishers, Dordrecht, 2004).

2. J.P. Coleman, L.Gr. Ixaru, Truncation errors in exponential fitting for oscillatory problems, SINUM 44 (2006) 1441-1465.

3. J.P. Coleman, L. Gr. Ixaru, P-Stability and exponential fitting methods for $y^{\prime \prime}=f(x, y)$, IMA Journal on Numerical Analysis 14 (1994) 1-21.

4. L. Gr. Ixaru, Numerical Operations on Oscillatory Functions. Computers \& Chemistry 25 (2001) 39-53.

5. http://www.ma.ic.ac.uk/jcash/BVP_software/PROBLEMS.PDF. 
Copyright of AIP Conference Proceedings is the property of American Institute of Physics and its content may not be copied or emailed to multiple sites or posted to a listserv without the copyright holder's express written permission. However, users may print, download, or email articles for individual use. 